\title{
Pre-Processing Method for Extraction of Pectoral Muscle and Removal of Artefacts in Mammogram
}

\author{
${ }^{1}$ Ojo J. A., ${ }^{2}$ Adepoju T. M., Omdiora E. O., Olabiyisi O. S. and Bello O. T. \\ ${ }^{1}$ Department of Electronic and Electrical Engineering, LAUTECH, Nigeria. \\ ${ }^{2}$ Department of Computer Science and Engineering, LAUTECH, Nigeria.
}

\begin{abstract}
Mammography is an effective imaging modality used by radiologists for detection of breast cancer and effective suppression of pectoral muscle as well as removal of noise in form of artefacts aids accurate detection. In existing methods, either over-segmentation or under-segmentation, have been reported resulting in less accurate results. In this work, an algorithm was developed that effectively removed artefacts and pectoral muscle from mammograms, it uses a region description method, which uses splitting and merging technique to extract pectoral muscle and remove unwanted noise from the original mammograms. Digital mammograms from MIAS database were used to test the algorithm. The algorithm achieved $92.24 \%$ good results,4.04\% acceptable results, $3.10 \%$ unaccepted results, and $0.62 \%$ of the mammograms were discarded. Experimental results obtained indicate that the breast regions extracted accurately.
\end{abstract}

Keywords: Mammograms, Pre-processing, Region of Interest (ROI).

\section{Introduction}

Pectoral muscles represent a predominant density region in the most medio-lateral oblique (MLO) views of mammogram and it appears more brightly in the mammogram [1]. The border between the fibroglandular region and the pectoral muscle is fuzzy and irregular because of noise and surrounding breast tissue. The gray level of the pectoral muscle is similar to that of abnormality in breast; therefore, there is need to remove such to avoid an increase in false detection rate at a later stage especially in the MLO view, where the pectoral muscle in the mammogram is slightly brighter compared with the rest of the breast tissue. Noisy or corrupted mammograms could lead to wrong diagnosis, therefore, the need to improve the quality of mammograms [2]. In addition, adequate removal of pectoral muscles and artefacts helps to limit the search for abnormalities without undue influence from background of the mammogram [3].

\section{Related Works}

Previous works on automatic detection of abnormalities in breast tissue from mammograms substantiate the fact that extraction of discriminative features may be affected by the presence of the pectoral muscle. Mammographic parenchyma and pectoral muscle have similar textural characteristics, causing a high number of false positives, during detection of suspicious abnormalities [4]. Multiple linked self organizing neural networks was used to segment mammogram into four components: background, pectoral muscle, fibroglandular tissue and adipose tissue [5]. Mammographic Image Analysis and System (MIAS) database is the common database used for testing algorithm for detection of breast cancer and most of the consist of a black background with significant noise [6]. Segmentation of region of interest in [6] was performed using the logarithm of the pixel energy and a region growing algorithm based on vertical and horizontal gradient and morphological operation. It was ascertained that sometimes location of mass is close to pectoral muscle in the images and a non-rigid threshold was employed for effective mass detection. Active contour technique was used to segment pectoral muscle and localised dense tissues using maxima method [7]. Hough Transformation is a widely used technique for detecting geometrical features in images [8]. Images are mapped into a parametric space which makes identification of particular geometrical features easier. A straight-line that represents the boundary of pectoral muscle in the mammogram images detected achieved $57.76 \%, 28.88 \%$ and $13.35 \%$ as good, acceptable and unacceptable results respectively, while two of the whole mammograms were discarded.

\section{Extraction Of Region Of Interest}

This paper employed region description method, which measures the properties of image region such as: area, perimeter, shape compactness and polygon approximation to suppress pectoral muscle, extract high intensity noise and artefacts. It measures a set of properties for each connected component (object) in the images. Area of a shape is number of pixels in a region. The area of a region $\mathrm{R}$ can be described using equation (1): 


$$
A(R)=\sum_{x} \sum_{y} f(x, y)
$$

where $f(x, y)=1$ on the pixels belonging to the region and zero otherwise. Perimeter is the boundary length. The perimeter of a region $\mathrm{R}$ is described in equation (2):

$$
P(R)=\sum_{i} \sqrt{\left(x_{i}-x_{i+1}\right)^{2}+\left(y_{i}-y_{i+1}\right)^{2}}
$$

where $\left(x_{i}, y_{i}\right)$ are the coordinates of the $i-t h$ pixel of the boundary.

Compactness is ratio of perimeter to area of a region i.e. $\left(\frac{\text { perimeter }^{2}}{\text { area }}\right)$. It is defined by equation (3):

$C(R)=\frac{4 \pi A(R)}{P^{2}(R)}=\frac{A(R)}{P^{2}(R) / 4 \pi}$

The denominator is the area of a circle whose perimeter is $P(R)$. It measures the ratio of area of the shape and the circle that can be traced with the same perimeter. Image mask multiplication was performed using equations (4) and (5) leading to extraction of the breast region. The convolution mask $=(r, c)$ and the image $I=(r, c)$, the convolution equation is given in equation:

$$
\begin{aligned}
& \sum_{x=-\infty}^{\infty} \sum_{y=-\infty}^{\infty} I(r-x, c-y) M(x, y) \\
& N_{x, y}=w_{0} \times O_{x-1, y-1}+w_{1} \times O_{x, y-1}+w_{2} \times O_{x+1, y-1}+w_{3} \times O_{x-1, y}+w_{4} \times O_{x, y}+w_{5} \times O_{x+1, y}+w_{6} \times O_{x-1, y+1}+w_{7} \times O_{x, y+1}+w_{8} \times O_{x+1, y+1} \\
& \forall_{x, y} \in 2, N-1
\end{aligned}
$$

where $\mathrm{N}$ is the new image.

For the $3 \times 3$ neighbourhood, nine weighty coefficients $w_{i}$ were applied the original image to calculate the value in new image $\mathrm{N}$ at point with co-ordinate $(x, y)$.

The extracted breast region of interest was filtered out using global thresholding technique. A threshold was set, after several tests, to separate adjacent regions of pectoral muscle distinguishable by intensity from the breast. A polygon approximation method (splitting technique) was employed to extract the pectoral region of the images.

\section{Discussion of Result}

The developed algorithm was tested on 322 images of mini-MIAS database to suppress pectoral muscle and remove the artefacts. The results obtained were shown in Table 1 . The evaluation was done subjectively through inspection and comparison with expert report contained in the readme file of the MIAS database. Images are termed 'good' if pectoral muscle is correctly suppressed and artefacts satisfactorily removed, an example is shown in Figure 1. 'Accepted' images are those that have a small portion of the pectoral muscle segmented with the breast region while artefacts are satisfactorily removed (over-segmentation), example in Figure 2. 'Unaccepted' images are those that have salient points that could reveal abnormalities were suppressed along with pectoral muscle (under-segmentation), example in Figure 3. 'Discarded' images are those in which pectoral muscle suppression and artefacts removal are poorly done, example in Figure 4. The new algorithm performed better when compared with an earlier work [8], where $57.76 \%$ good, $28.88 \%$ acceptable, $13.35 \%$ unacceptable and $0.62 \%$ discarded were results achieved.

Table 1: Pre-process Results Evaluation

\begin{tabular}{lll}
\hline Subjective evaluation & Result & Percentage (\%) \\
\hline Good & 297 & 92.24 \\
Accepted & 13 & 4.04 \\
Unaccepted & 10 & 3.10 \\
Discarded & 2 & 0.62 \\
\hline
\end{tabular}




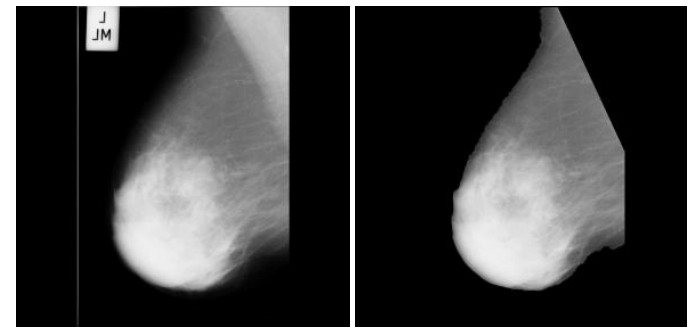

Figure 1: (a) Original (b) Good

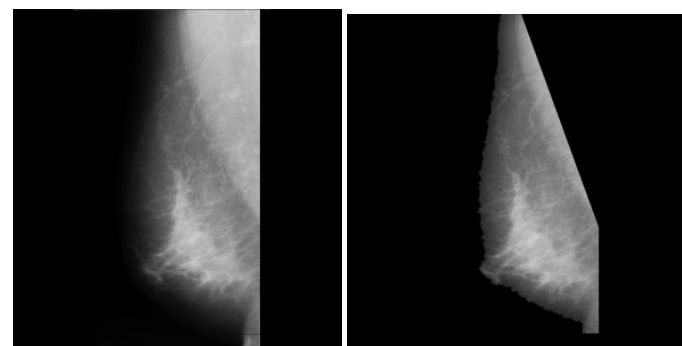

Figure 2: (a) Origina $\quad$ (b) Accepted

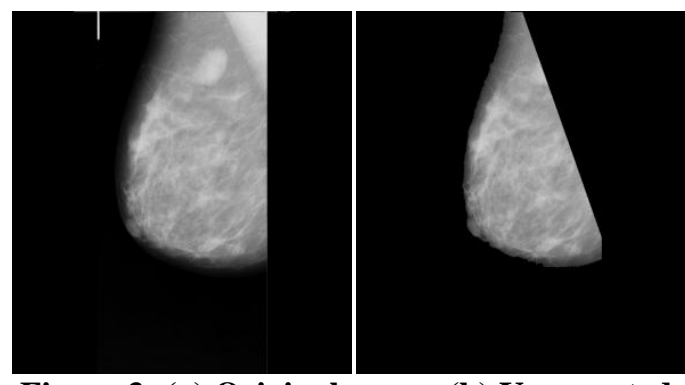

Figure 3: (a) Original $\quad$ (b) Unaccepted

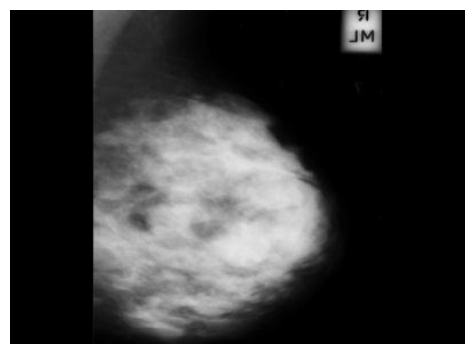

Figure 4: Discarded image

\section{Conclusion}

A combination of region description and polygon approximation methods to suppress pectoral muscle, extract the high intensity noise and artefacts has been presented in this paper. The results of the developed algorithm enhanced the pre-processed images, consequently reducing false positives in detection of breast cancer in mammograms.

\section{References}

[1] Boucher, A., Jouve, P.E., Cloppet, F. and Vincent, N., Segmentation du muscle pectoral sur une mammographie, Congrès des jeunes chercheurs en vision par ordinateur, ORASIS'09, 2009, Trégastel, France. 
[2] Ponraj, D.Narain, Jenifer, M.Evangelin Poongodi, P., and Manoharan, J.Samuel, A Survey on the Preprocessing Techniques of Mammogram for the Detection of Breast Cancer, Journal of Emerging Trends in Computing and Information Sciences, 2(12), 2011, ISSN 2079-8407.

[3] Bandyopadhyay, Kumar, Samir, pre-processing of Mammogram Images, International Journal of Engineering Science and Technology, 2(11), 2010, 6753-6758.

[4] Gupta, R., and Undril, P.E., The use of texture analysis to delineate suspicious masses in mammography”, Phys.Med.biol. 40, 1995, $835-855$

[5] Suckling, J., Dance, D., Moskovic, E., Lewis, D. and Blacker, S., Segmentation of mammograms using multiple linked self-organizing neural networks", Med. Phys, 22, 1995, 145-52.

[6] Moayedi, F., Boostani, R., Kazemi, A. R., Katebi, S. and Dashti, E., Subclass Fuzzy-SVM Classifier as an Efficient Method to Enhance the Mass Detection, Mammograms Iranian Journal Of Fuzzy Systems, 7 (1), 2010, 15-31.

[7] Eddaoudi, Fatima, Regragui, Fakhita Mahmoudi, Abdelhak Lamouri, and Najib, Masses Detection Using SVM Classifier Based on Textures Analysis, "Applied Mathematical Sciences", 5(8), 2011, 367 - 379

[8] Tomàs, J. S. Segmentation of the breast region with pectoral muscle suppression and automatic breast density classification, A thesis submitted for the degree of Master Ingénieur Civil Électricien, 2011. 